\title{
Use of Millimeter Wave FMCW Radar to Capture Gait Parameters
}

\author{
Hajar Abedi*, George Shaker, Jennifer Boger, Plinio Morita and Alexander Wong \\ University of Waterloo, Waterloo, Ontario, Canada \\ *Corresponding author: : Hajar Abedi, University of Waterloo, Waterloo, Ontario, Canada \\ To Cite This Article: Hajar Abedi. Use of Millimeter Wave FMCW Radar to Capture Gait Parameters. Am J Biomed Sci \& Res. 2019 - 6(2). AJBSR. \\ MS.ID.001009. DOI: 10.34297/AJBSR.2019.06.001009.
}

Received: 眥 November 01, 2019; Published: 眥 November 12, 2019

\begin{abstract}
Features related to gait can be fundamental metrics of human motion. Gait speed and stride length are increasingly being recognized as a measure a person's health status; changes in these parameters can indicate changes in health. However, variations in gait characteristics as a result of cognitive or other conditions may go undetected as the effect can be gradual and often goes unnoticed during clinic visits. Technologies that detect changes in the gait of older adults could support detection, evaluation, and monitoring of parameters related to changes in mobility, cognition, and frailty. This, in turn, could be used to support more effective biomedical applications. To do so, we must be able to extract crucial gait parameters with high accuracy while maintaining ease of use for practical implementation. This paper describes the development of a millimeter wave FMCW (frequency modulated continuous wave) radar sensor that can measure walking speed and stride length in range of indoor environments. Since our approach uses noninvasive wireless technologies, people do not need to wear or carry a device on their body. Additionally, due to the fact that there is no camera, feelings of privacy are more readily supported compared to videobased methods.
\end{abstract}

\section{Introduction}

Despite gait cycles appearing to be relatively simple, an indepth analysis of the unique features and patterns generated by an individual can give valuable biodata that can be used to enhance patient care. Through the analysis of average gait velocity, rhythm, as well as any abnormalities, gait cycle can indicate musculoskeletal fitness, cardiovascular health, mental wellness, and even the expected mortality [1], [2]. The data collection methods must be precise in order to correctly diagnose a variety of latent conditions that can later be clinically addressed by health professionals through gradual adjustments. The most commonly available gait feature and motion capturing methods include force mats, wearable sensors and video camera systems. The crucial factors that must be examined for each method include patient comfort, practicality, expense, precision, and portability for ideal implementation into healthcare. Force plates embedded into a mat can observe the body mass and changes in pressure output during a series of steps to output velocity, stride length as well as force distribution on the feet during moments of the gait cycle [3]. GAITRite, a portable single layer pressure sensitive walkway mat, has proven to be easy to use and very reliable for determining step asymmetry and stride lengths for various walking speeds [4]. However, the usefulness of the gait analysis on patients with documented disease, physical injury, and young children need to be further examined as currently there is not enough data supporting such use. The length of the mat is another challenge which would restrict the time duration for gait assessment. Additionally, GAITRite systems incur substantial overhead cost. Similar limitations also apply to video camera system. In video camera-based systems, methods such as the interval particle filtering algorithm could be implemented for a comprehensive motion analysis [5]. However, video camera systems also have significant limitations, as for example difficulty in constant tracking of the individual when performing tasks other than straight line walking such as sitting or turning. Another potential challenge would be parts of the body crucial for gait analysis that are in blind spots to the camera. Using multiple cameras could be a potential solution, but would dramatically decrease the portability, and significantly increase the cost as well as the potential for gait analysis errors. Additionally, camera-based systems can lead to significant privacy issues. Lastly, while wearable sensors are less expensive and much more accurate [6], individuals are required to wear or carry a device on their body, which is not ideal for long term monitoring. Radar sensors could be one promising technique which could be used to monitor gait parameters frequently [7], [8]. In this paper we use a low-cost, low power FMCW radar to acquire gait speed and stride length. Since the radar sensor is a non- 
invasive technology, propagating electromagnetic waves, people do not need to wear or carry a device on their body.

\section{Procedure and Measurement Results}

As one of the most promising among other radar techniques, our team chose to use FMCW radar in the $79 \mathrm{GHz}$ frequency band. Using FMCW. We can obtain linear combination of sinusoid signals with low frequencies by multiplying the received signal by the transmitted signal. Therefore, FMCW radar systems can enable simpler hardware and architecture types and provide information about the range and radial velocity of multiple targets by processing the observations of beat signals [9]. In our study, we asked our participants to walk a $5 \mathrm{~m}$ section, which was repeated 6 times. Data was collected using the selected radar and was followed by analysis of the corresponding gait parameters. In order to obtain information about walking speed, we first applied Fast Fourier Transform (FFT) on received signal. Based on the information of the position of the target (e.g. a person walking) and the time, we can easily calculate walking speed. Fig.1 (a) shows the trajectory of a person waking in front of the radar. As depicted, we can see the distance to the radar increases when the person moving away from the radar and decreases when the person moves toward the radar. In one test case, post-processing the result showed that the walking speed was $0.98 \mathrm{~m} / \mathrm{s}$ while the real speed was around $1 \mathrm{~m} / \mathrm{s}$. In order to get the stride length, we needed information about the motion of other parts of the body, since the maximum reflection changes between torso and legs during walking. When we walk, different parts of our body move at different velocities, which leads the micro-Doppler effect. This effects can be calculated using a joint time-frequency analysis [10]. We can then use a Short-Time Fourier Transform (STFT) to calculate the spectrogram of the backscattered signal and visualize the micro-Doppler effect of a person walking. The spectrogram shown in Figure 1(b) demonstrates an example of the overall micro-Doppler effects of walking obtained by our FMCW radar sensor.

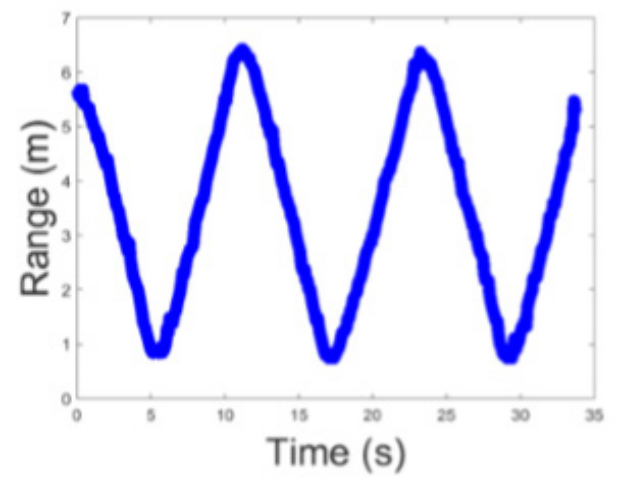

(a)

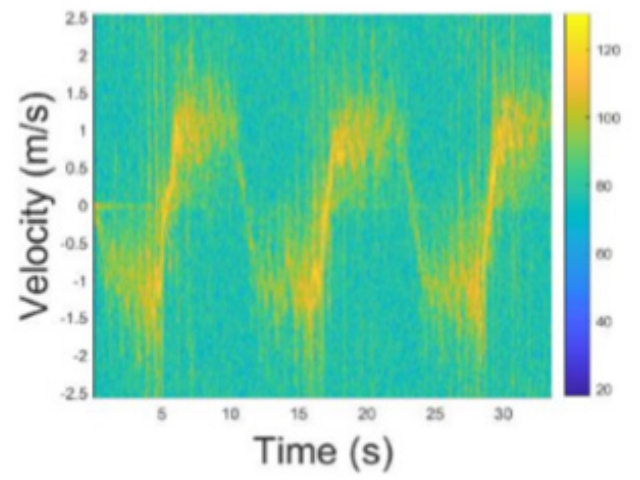

(b)

Figure 1: FMCW radar results of (a) trajectory of walking and (b) STFT method.

\section{Conclusion}

In this paper, we explore gait parameters measurement with a low-cost, mm-wave FMCW radar. We showed walking speed and step length could be obtained by FMCW radar. Compared with other gait measurement technologies, such as GatiRite, radar is affordable which could be used at home. Moreover, there is no need for physicians participants which makes the radar-based gait monitoring a zero-effort technology. Our future purpose is to use the proposed radar technology for fall detection and gait monitoring for multiple people environment.

\section{References}

1. M Quan (2017) "Walking Pace and the Risk of Cognitive Decline and Dementia in Elderly Populations: A Metaanalysis of Prospective Cohort Studies." J Gerontol A Biol Sci Med Sci72(2): 266-270.

2. J Afilalo (2010) "Gait speed as an incremental predictor of mortality and major morbidity in elderly patients undergoing cardiac surgery." J Am Coll Cardiol 56(20): 1668-1676.

3. Anonymous. The Science of Force Plates and Pressure Mapping.
4. AL McDonough (2001) "The validity and reliability of the GAITRite system's measurements: A preliminary evaluation." Arch Phys Med Rehabil 82(3): 419-425.

5. J Saboune and F Charpillet (2005) "Using interval particle filtering for marker less 3D human motion capture".

6. W Tao (2012) "Gait analysis using wearable sensors." Sensors (Basel) 12(2): 2255-2283.

7. PP Morita (2019) "Comparison of Gait Speed Estimation of Multiple Sensor-Based Technologies." Proceedings of the International Symposium on Human Factors and Ergonomics in Health Care 8(1): 135-139.

8. A Boroomand (2018) "Autonomous gait speed estimation using $24 \mathrm{GHz}$ FMCW radar technology. 2018 IEEE EMBS International Conference on Biomedical \& Health Informatics (BHI) pp. 66-69.

9. B Kim (2019) "A Low-Complexity FMCW Surveillance Radar Algorithm Using Two Random Beat Signals." Sensors (Basel, Switzerland) 19(3): 608.

10. Victor C Chen (2014) "Anonymous Radar Micro-Doppler Signatures: Processing and Applications. 